ARTICLE

DOI: $10.1038 / \mathrm{s} 41467-017-02212-w$

\title{
CTCF driven TERRA transcription facilitates completion of telomere DNA replication
}

\author{
Kate Beishline ${ }^{1,2}$, Olga Vladimirova ${ }^{1}$, Stephen Tutton ${ }^{1}$, Zhuo Wang (D) $^{1}$, Zhong Deng ${ }^{1}$ \& Paul M. Lieberman ${ }^{1}$
}

Telomere repeat DNA forms a nucleo-protein structure that can obstruct chromosomal DNA replication, especially under conditions of replication stress. Transcription of telomere repeats can initiate at subtelomeric CTCF-binding sites to generate telomere repeat-encoding RNA (TERRA), but the role of transcription, CTCF, and TERRA in telomere replication is not known. Here, we have used CRISPR/Cas9 gene editing to mutate CTCF-binding sites at the putative start site of TERRA transcripts for a class of subtelomeres. Under replication stress, telomeres lacking CTCF-driven TERRA exhibit sister-telomere loss and upon entry into mitosis, exhibit the formation of ultra-fine anaphase bridges and micronuclei. Importantly, these phenotypes could be rescued by the forced transcription of TERRA independent of CTCF binding. Our findings indicate that subtelomeric CTCF facilitates telomeric DNA replication by promoting TERRA transcription. Our findings also demonstrate that CTCFdriven TERRA transcription acts in cis to facilitate telomere repeat replication and chromosome stability.

\footnotetext{
${ }^{1}$ The Wistar Institute, Philadelphia, PA 19104, USA. ${ }^{2}$ Present address: Department of Biology and Allied Health Sciences, Bloomsburg University, Bloomsburg, PA 17815, USA. Correspondence and requests for materials should be addressed to K.B. (email: kbeishline@bloomu.edu)

or to P. M.L. (email: Lieberman@wistar.org)
} 
$\mathrm{R}$ eplication and maintenance of telomere repeat DNA is a major challenge to rapidly dividing cells ${ }^{1-3}$. Telomere repeats are bound by a multi-factor complex known as Shelterin that binds and protects telomeres throughout the cell cycle and maintains the double-strand and single-strand portions of the repeat sequences 4,5 . Shelterins have well-characterized roles in regulating telomere repeat length homeostasis and DNA damage signaling. Adjacent to the TTAGGG tandem repeats are variable repetitive regions known as the subtelomeres ${ }^{6,7}$. Subtelomeric sequences are bound by various nuclear factors that have been implicated in the regulation of transcription and chromatin structures important for telomere protection and regulation $^{8-12}$.

One major function of the subtelomere sequences is to regulate the transcription of the telomere repeat-encoding RNA (TERRA). TERRA transcripts have been identified in multiple organisms, including yeast and human, and are known to initiate within subtelomeric repeat regions and proceed into the terminal ${\text { (TTAGGG })_{n} \text { repeats }}^{13-15}$. The function of these transcripts has been addressed in multiple studies, but it remains unclear whether these transcripts function directly on the telomeres from which they are transcribed (cis-acting) or whether they work remotely (trans-acting) on other telomeres or locations. Studies suggest that TERRA can bind both in cis to the telomere from which it is produced $^{16,17}$ as well as from a cellular pool that can interact in trans with other TERRA producing or non-producing chromosome ends ${ }^{18,19}$. Transcribed TERRA has been shown to interact with the telomere DNA either through direct interaction and the formation of R-loops, or through interaction with RNA binding factors which recruit TERRA to telomeric chromatin ${ }^{16,20-26}$. Other studies have shown that TERRA transcript levels peak during $S$ phase, and further decrease as cells enter G2/M phase, suggesting that TERRA may function during cell replication ${ }^{27,28}$. Additionally, TERRA is highly overexpressed in cancer cells utilizing alternative lengthening of telomere (ALT) mechanisms, which lack telomerase, but have variable length telomere repeats replicated in part through homologous recombination $16,21,24,29-$ 31. All in all, the function of telomeric transcription and TERRA RNA in regulating telomere DNA replication, and whether it functions locally in cis or remotely in trans, remains unclear.

TERRA transcription initiates within the subtelomere and various regulatory elements have been implicated in its transcriptional control. The precise initiation site may vary for each chromosome, and a single telomere may be responsible for generating the majority of TERRA transcripts in some cells or organisms ${ }^{19}, 25$. A major initiating element for human TERRA was localized to a subtelomeric CpG-island ${ }^{32}$, which was found to contain high-affinity binding sites for CTCF and cohesin ${ }^{9}$. ChIPSeq analyses also revealed that RNA polymerase II was enriched at these CTCF-cohesin sites in a large percentage of human subtelomeres ${ }^{8,9}$. Depletion of CTCF and cohesin have resulted in alterations in TERRA transcription and telomere regulation, suggesting that TERRA transcription regulates telomere repeat stability. CTCF has many genomic functions, including the organization of higher-order $3 \mathrm{D}$ chromatin structures, nucleosome positioning, and regulation of RNA polymerase II initiation and RNA processing ${ }^{33,34}$. It is yet unknown how the many possible genomic functions of CTCF are contributing to TERRA regulation and telomere maintenance. Additionally, more direct evidence that TERRA transcription contributes to the stability of the transcribed source telomere has not yet been provided. In this study we address the regulation and function for TERRA by CRISPR/CAS9 gene editing of a subtelomeric CTCF-binding motif implicated in TERRA transcription initiation. We show that CTCF-dependent transcription of the $17 \mathrm{p}$ subtelomere is important for completion of replication of that telomere under stress, and in the absence of CTCF and TERRA transcription, these sites form ultra-fine anaphase bridges and micronuclei during and after mitosis, respectively.

\section{Results}

CRISPR/Cas9 targeting of 17p family of subtelomeric CTCFbinding sites. A series of highly conserved CTCF-binding motifs found on most subtelomeres within a few kilobases from the telomere repeats has been implicated in TERRA transcriptional regulation ${ }^{9,} 25$. We targeted the family of subtelomeric CTCFbinding sites represented by chromosome arm 17p, which is located in close proximity $(<5 \mathrm{~kb})$ to the (TTAGGG) $)_{\mathrm{n}}$ repeat junction, shows strong colocalization with CTCF and cohesin in ChIP assays, and is known to produce TERRA in multiple cell types $^{8,9}$ (Fig. 1a). The 17p family CTCF-binding sites consist of a more extensive homology region (up to $20 \mathrm{~kb}$ ) that is identical to that found at the terminal end of $7 \mathrm{p}, 9 \mathrm{q}, 3 \mathrm{q}$, as well as nonterminal subtelomere repeat regions on $16 \mathrm{q}$ and $11 \mathrm{p}$ (Fig. 1b). The 17p family CTCF-binding sites are composed of 2 tandem CTCF-binding motifs, oriented in the direction of the telomere end, separated by about $35 \mathrm{bp}$ of sequence. These CTCF sites are highly similar to those described previously to function at promoters and enhancers in transcriptional activation ${ }^{35}$. HCT116 cells were transfected with Cas9/gRNA constructs along with a homology block of DNA to promote repair of the chromosome target, replacing a $92 \mathrm{bp}$ fragment around the CTCF-binding motifs with a single BamHI site. The cells were clonally selected and verified by PCR amplification, restriction digest, and sequencing (Fig. 1c, d). We selected two HCT116 clones, one containing a small fraction of mutated sites (Mutant 1), while the other containing a larger proportion of mutated sites (Mutant 2) among the multiple members of the $17 \mathrm{p}$ family of CTCF sites. Mutations in Mutant 2 could be validated by Sanger sequencing (Fig. 1e), and generated a single uniform PCR product with a BamHI restriction site insertion. Control cell lines were selected either from clonal selection of cells expressing no guide RNA (control for wildtype 1), or clones selected during the mutation validation stage, which were not found to contain any mutant copies (control for wildtype 2).

CTCF-binding and H3K4me3 are lost at mutated CTCF sites. CTCF binding in wildtype and mutant cell lines was verified by chromatin immunoprecipitation (ChIP) (Fig. 2a, b). The most extensively mutated cell line (Mutant 2) showed the largest decreases in CTCF binding around the mutated binding site, while it maintained CTCF binding at the Xq family of subtelomeres, which were not targeted by CRISPR mutation (Fig. 2b). Histone $\mathrm{H} 3$ binding around $17 \mathrm{p}$ family sites was maintained, with a modest trend toward an increase binding at regions adjacent to the deletions (Fig. 2c). In contrast, histone $\mathrm{H} 3 \mathrm{~K} 4 \mathrm{me} 3$ levels were significantly decreased at sites adjacent to mutated CTCF-binding sites (Fig. 2d). Furthermore, mutant cells exhibited a decrease in TERRA expression of $17 \mathrm{p}$ family transcripts (including the $7 \mathrm{p}$ subfamily that contains mutated CTCFbinding sites), while other non-mutated chromosome TERRA transcripts (15q and $\mathrm{Xq}$ ) and global levels of TERRA transcripts showed no significant change (Fig. 2e; Supplementary Fig. 1a, b). We did not detect any changes in TRF2 binding to adjacent subtelomeric sequences at $17 \mathrm{p}$-mutated telomeres or at telomere repeat DNA (Supplementary Fig. 1c, d). These findings indicate that CRISPR/Cas9-mutated CTCF-binding sites on the 17p subtelomere family lead to a local loss of CTCF, histone H3K4me3, and decrease TERRA transcription, but do not disrupt global TERRA levels or TRF2 binding. 
a

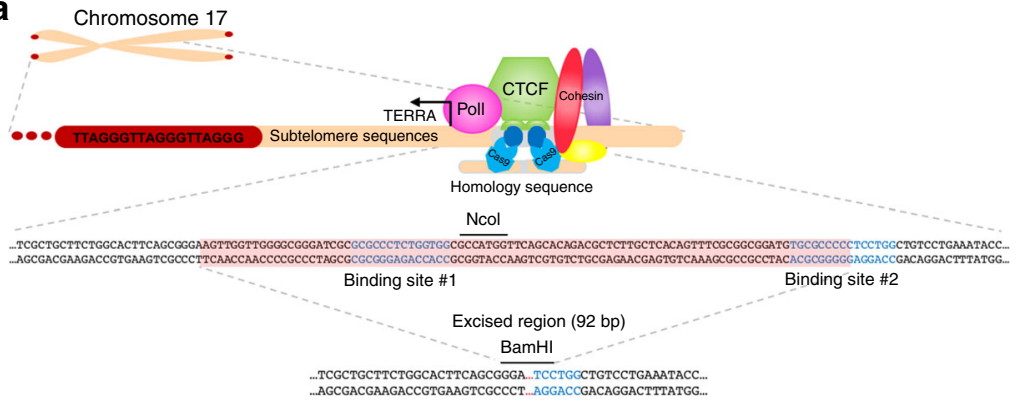

d

d Wildtype 2 Mutant 2 Mutant 1

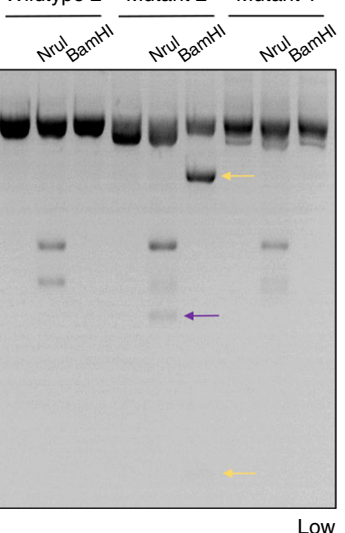

C

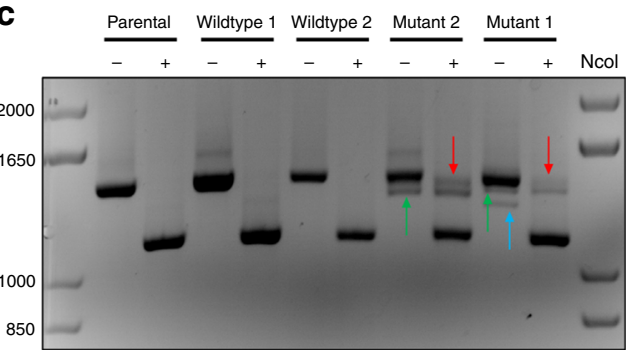

Low b
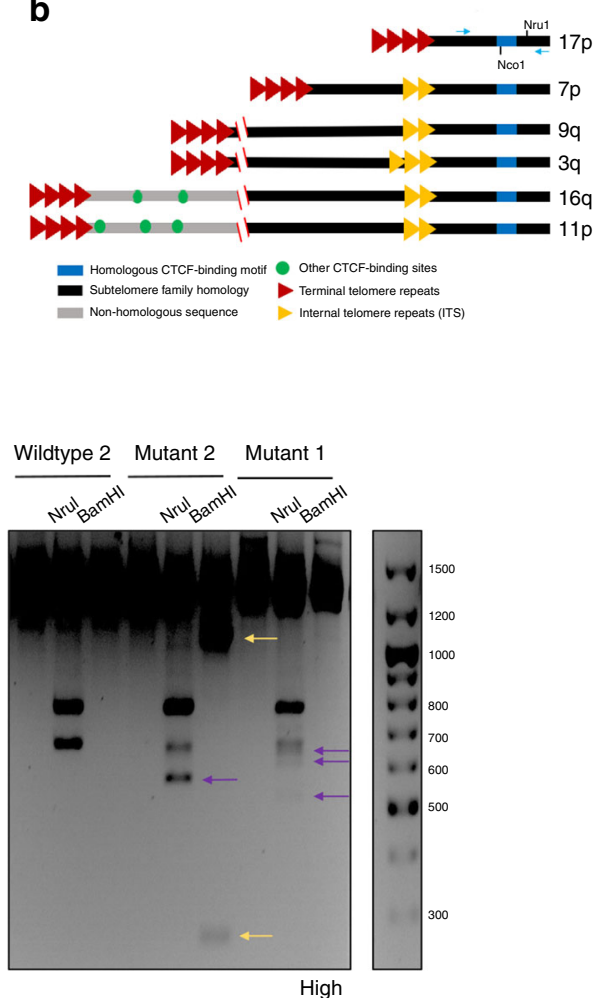

High

e

Subtel 17p 3860-3980 TCTGGCACTTCAGCGGGAAGTTGGTTGGGGCGGGATCGCGCGCCCTCTGGTGGCGCCATGGTTCAGCACAGACGCTCTTGCTCACAGTTTCGCGGCGGATGTGCGCCCCCTCCTGGCTGTC Wildtype 2 clone TCTGGCACTTCAGCGGGAAGTTGGTTGGGGCGGGATCGCGCGCCCTCTGGTGGCGCCATGGTTCAGCACAGACGCTCTTGCTCACAGTTTCGCGGCGGATGTGCGCCCCCTCCTGGCTGTC

Fig. 1 CRISPR-engineered mutants of CTCF 17p family subtelomere-binding sites. a CRISPR-Cas9 guide RNAs were designed to target regions around the CTCF-binding motif on the subtelomere of 17p. Homology-directed repair was promoted by co-transfection of a DNA fragment-containing homologous DNA sequences flanking a BamH1 site, created by deleting 92 bp base pairs of DNA surrounding the CTCF-binding motif. $\mathbf{b}$ Schematic depicting basic homology between 17p family of subtelomeres. c A 1400 bp fragment of DNA surrounding the deleted locus was PCR-amplified from genomic DNA collected from CRISPR clonal cell lines. PCR fragments from parental, or selected wildtype and mutant cells were undigested (-) or digested (+) with Ncol, a restriction site only present within the 92 bp deleted region. Undigested mutant fragments are indicated with red arrows. Green and blue arrows indicate mutant fragments of decreased size in undigested samples. DNA markers in first and last lanes. d Further digests with Nrul and BamHI were utilized to identify mutant fragments. Nrul digests outside the excised region only on 17p and 11p and generates two bands, $640 / 760$ bp in wildtype and 548/760 bp in mutants. Purple arrows indicate bands created by mutant fragments. The BamHI cut site is introduced in clones that are repaired by perfect homologous recombination using the supplied gene block. Digestions creates 2 bands from mutant fragments, at 246 bp and 1063 bp, indicated by yellow arrows. Low (left) or high (right) image exposure. e Sanger DNA sequence validation of PCR fragment from Mutant 2 compared to wildtype clone and 17p consensus sequence

17p CTCF site mutants exhibit sister-telomere loss under replication stress. We next determined the effect of CTCFbinding site mutation on telomere integrity. Southern blot telomere-length assays revealed that bulk telomere length for mutant 2 was slightly greater than wildtype clone and parental cells (Supplementary Fig. 1f, g). However, this loss may be due to clonal variation, as the telomere length remained stable and did not continue to shorten in mutant cell lines. Analysis of metaphase spreads from thymidine synchronized cells revealed a significant increase in sister-telomere loss in mutant cell lines $($ Fig. $3 a-c)$. These effects were specifically enriched at $17 \mathrm{p}$ telomeres as determined by dual FISH using FISH probes specific for C-strand telomere repeat (red) and $17 \mathrm{p}$ subtelomeric DNA (green) (Fig. 3a, b). Sister-telomere signal loss was also observed using a G-strand telomere probe (Supplementary Fig. 2a), indicating that there is no strand specificity to sister-telomere loss. Sister-telomere loss was not observed at the distal $17 \mathrm{q}$ arm of mutant cell lines (Fig. 3b, right panel), indicating that the effect occurs proximal to the deletion of subtelomeric CTCF. We did not observe any significant differences in signal-free ends where both sister signals are lost (Supplementary Fig. 2b-d).
Interestingly, the loss of sister-telomere signal was not observed in metaphase spreads from cells that were not pre-synchronized with double thymidine block (Fig. 3c). As double thymidine treatment can lead to replication stress, it is possible that subtelomeric CTCF facilitates sister-telomere replication especially under conditions of replication stress.

17p CTCF site mutants exhibit decreased replication under stress. To test whether mutation of subtelomeric CTCF-altered DNA replication, we measured the levels of BrdU incorporation by BrdU-immunoprecipitation assay at sites adjacent to subtelomeric region of $17 \mathrm{p}$ in wildtype vs. mutant cells. Cells were synchronized by double thymidine block and released, followed by incorporation of BrdU during $S$ phase. Replicated DNA was assessed by BrdU IP followed by qPCR. Mutant cells showed a significant loss of BrdU incorporation during $S$ phase at subtelomeric regions closest to the $17 \mathrm{p}$ family telomere repeats compared to wildtype cells (Fig. 3d), a difference that diminished with distance from the repeats. No difference in BrdU incorporation was seen at other telomere adjacent loci. Flow cytometry 
a

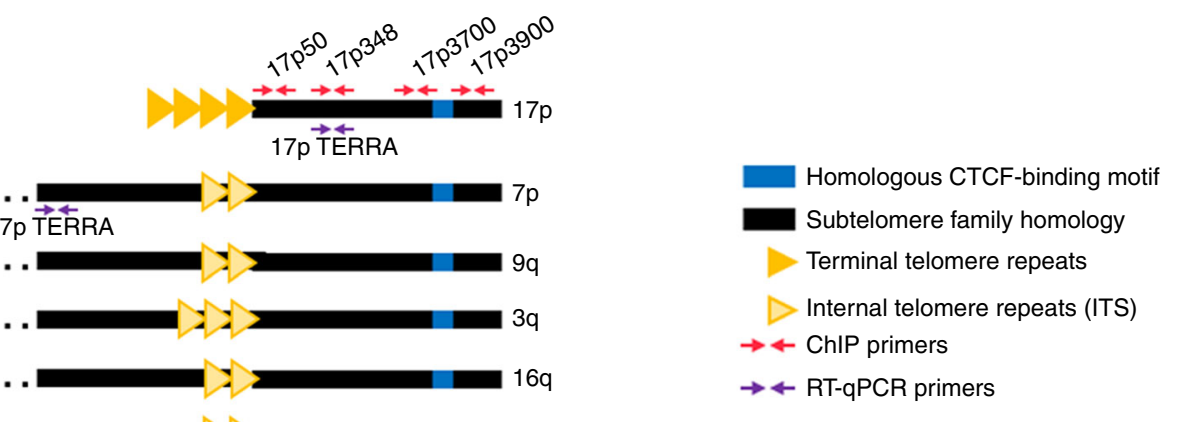

b
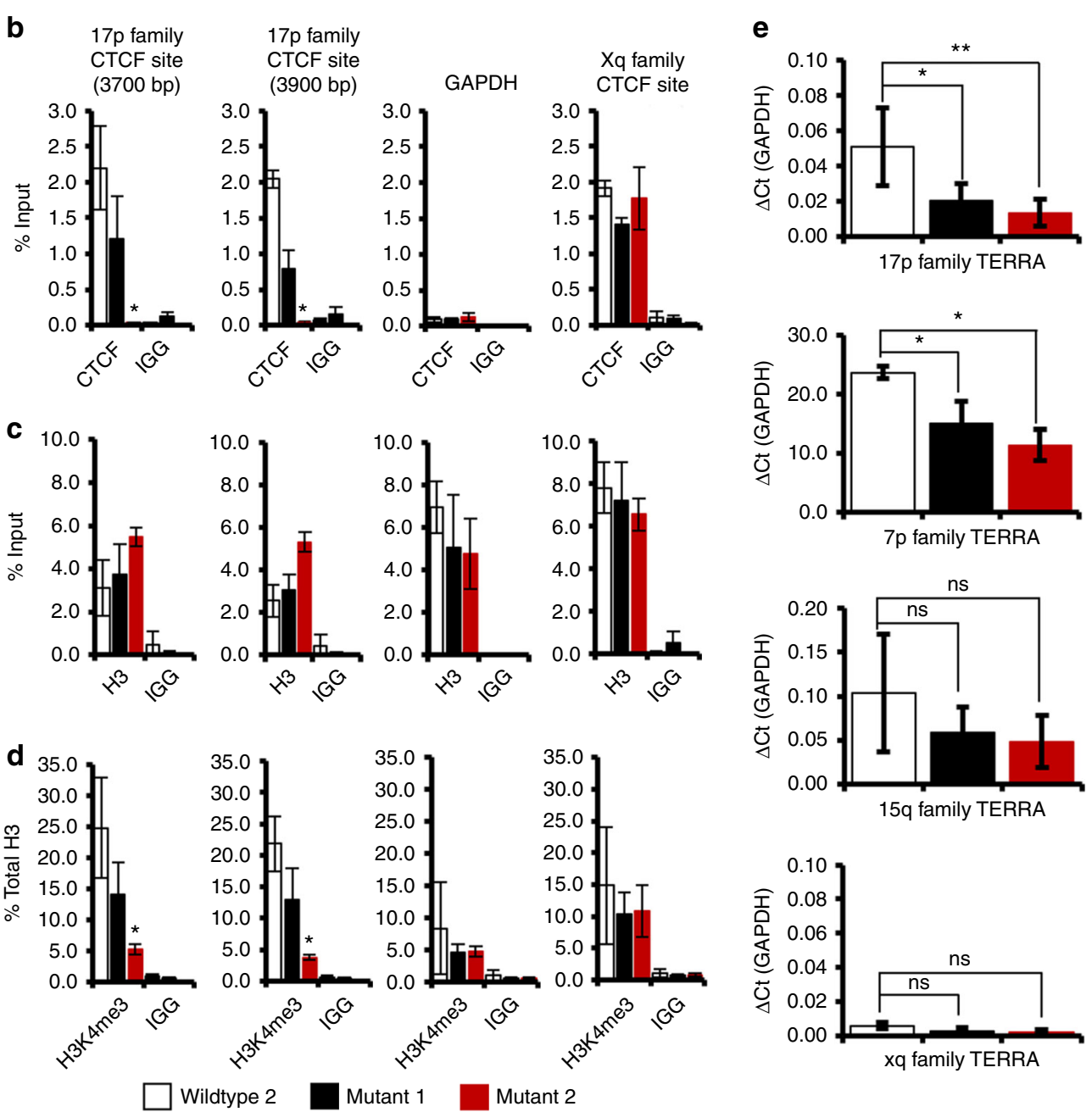

Fig. 2 CTCF site-deletion mutants exhibit local decreases of subtelomeric CTCF, H3K4me3, and TERRA transcription. a Schematic of 17p family and 7p subfamily homology with position of primers used for RT-qPCR (purple) and ChIP-qPCR (red). b Wildtype (white bar) and mutant cells (black and red bars) were collected and chromatin immunoprecipitation was performed to look at binding of CTCF; c total histone H3; d histone H3K4me3 at either 17p family CTCF sites (primer set at 3700 bp relative or at 3900 bp relative to telomere repeat tracks), GAPDH, or the Xq family of subtelomeric CTCF sites. e RNA was isolated from cell lines and analyzed by RT-qPCR to assess chromosome-specific TERRA transcription levels for primers detecting all 17p family TERRA, 7p family, 15q family, or Xq family TERRA transcripts. All ChIP and RNA experiments represent 3-5 combined biological replicates with error bars expressing SEM of replicates. ${ }^{\star} p<0.05 ;{ }^{\star \star} p<0.01$, or not significant (ns), measured by Student's $t$ test

indicated that the cells cycled at the same rate after treatment (Supplementary Fig. 3a) and a time course of BrdU incorporation at the loci closest to the $17 \mathrm{p}$ telomere end as well as other subtelomere ends showed no significant change in the rate of BrdU incorporation between mutant and wildtype cells (Supplementary Fig. 3b). These data suggest that subtelomeric CTCF-binding site at $17 \mathrm{p}$ is required for the completion of local DNA replication, but does not change the overall rate or genome-wide DNA replication.

17p CTCF site mutants exhibit ultra-fine anaphase bridges. Several studies demonstrate that replication stress can lead to the formation of ultra-fine anaphase bridges ${ }^{36,}{ }^{37}$. Ultra-fine 


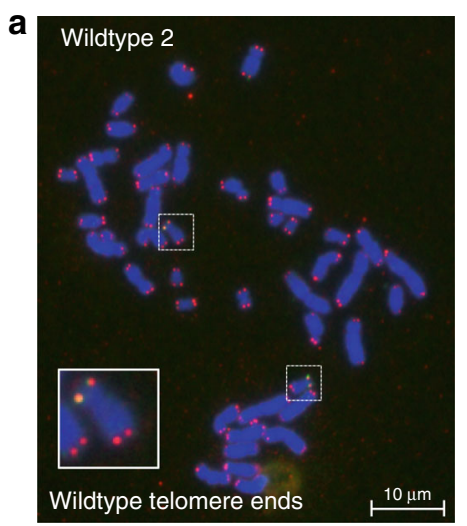

b

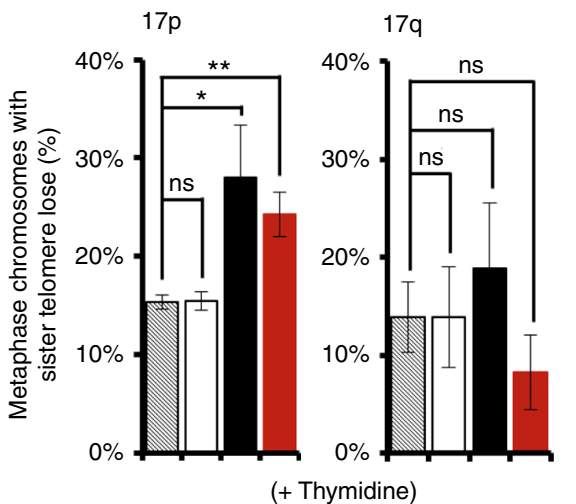

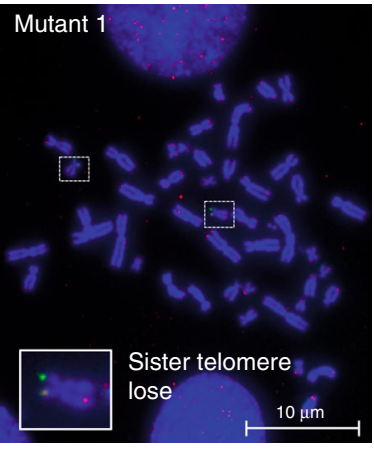

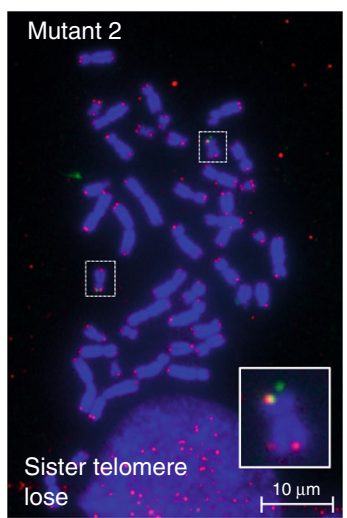

C

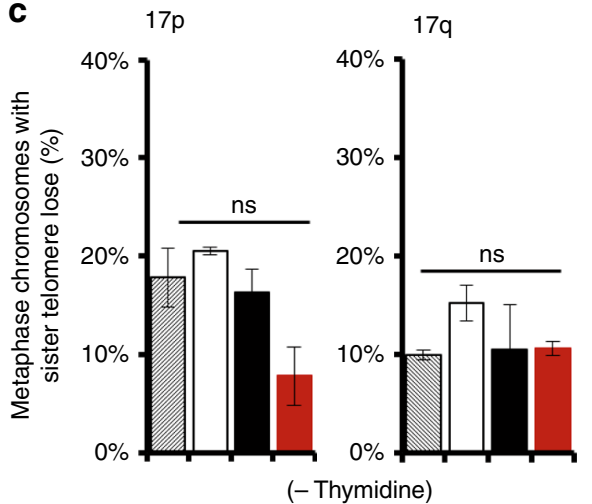

d
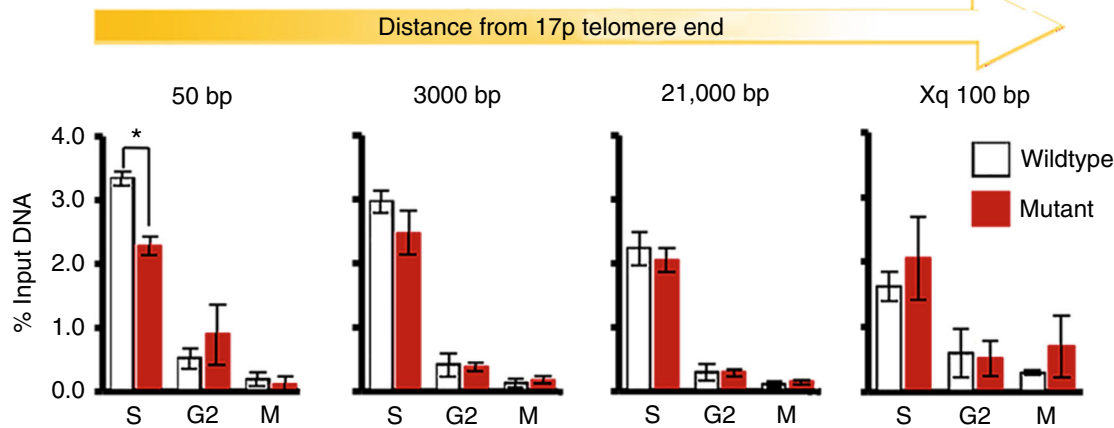

Fig. 3 CTCF site mutant 17p telomere ends exhibit mitotic sister-telomere loss and decreased replication. a Cells were synchronized by double thymidine block, released for $7 \mathrm{~h}$, colcemid was added, and mitotic cells were collected $1 \mathrm{~h}$ later. Representative images of mitotic spreads in wildtype 2 , Mutant 1 , or Mutant 2 clones stained with dual FISH for telomere repeats (red) and 17p subtelomeric sequences (green). Left, wildtype; Center, 17p Mutant 1; Right, 17p Mutant 2. b, c Quantification of sister-telomere loss on chromosome 17, p and q arms in cells synchronized with double thymidine block (+thymidine) (b), or asynchronous cells (-thymidine) (c). Data represented as average of 3 biological replicates, $n=150-200$ metaphases, error bars represent SEM. ${ }^{\star} p<$ $0.05 ;{ }^{\star \star} p<0.01$, measured by Student's $t$ test. d Cells were synchronized and released in the presence of BrdU either during the first $6 \mathrm{~h}$, for $\mathrm{S}$ phase or

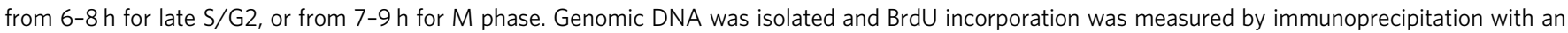
anti-BrdU antibody. Data represents average of three independent experiments with error in SEM

anaphase bridges (UFBs) are thought to form due to the presence of un-replicated regions or those which have not been decatenated prior to anaphase $\mathrm{s}^{36,38}$. To determine whether telomeres lacking subtelomeric CTCF-binding sites formed ultra-fine anaphase bridges, we analyzed the CTCF-deleted chromosome arm at $17 \mathrm{p}$ through metaphase and into anaphase. Anaphase preparations of synchronized cells were stained for PLK1-interacting checkpoint helicase (PICH), a DNA helicase known to bind to ultra-fine anaphase bridges ${ }^{38}$. We found mutant cell lines had an increased number of PICH-positive UFBs during mitosis (Fig. 4a, b; Supplementary Fig. 4a). When we co-stained anaphase cells with $\mathrm{PICH}$ and $17 \mathrm{p}$ FISH, we found a significant enrichment of the $17 \mathrm{p}$ chromosome arm on or flanking PICH bridges in mutant cells while $17 \mathrm{p}$ presence near PICH bridges in wildtype cells was extremely rare (Fig. 4c-e; Supplementary Fig. 4a). These findings suggest that subtelomeric CTCF functions locally and in cis to facilitate the completion of telomere DNA replication.

Sister-telomere loss in the presence of thymidine block, as well as formation of UFBs at the $17 \mathrm{p}$ telomere end suggested a possible sensitivity of the mutant cells to replication stress. We treated cells with another common replication stress-inducing agent aphidicolin to test for a similar sensitivity. We saw similar trends in UFB formation in aphidicolin-treated mutant cells as those we saw with thymidine treatment (Supplementary Fig. 4b, 
a
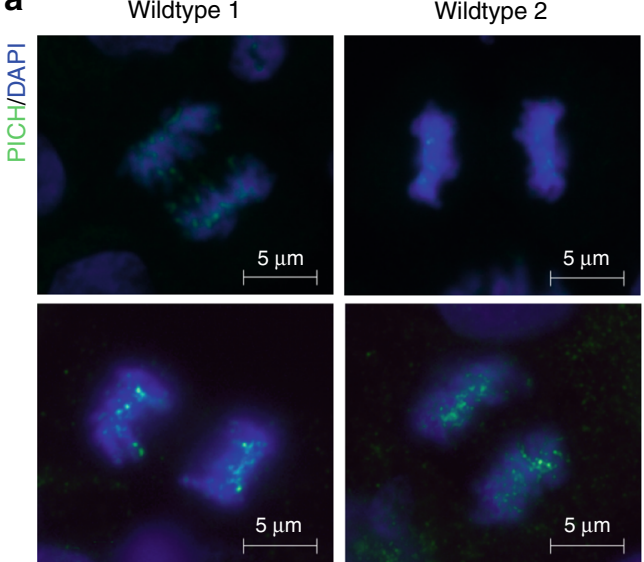

Wildtype 2
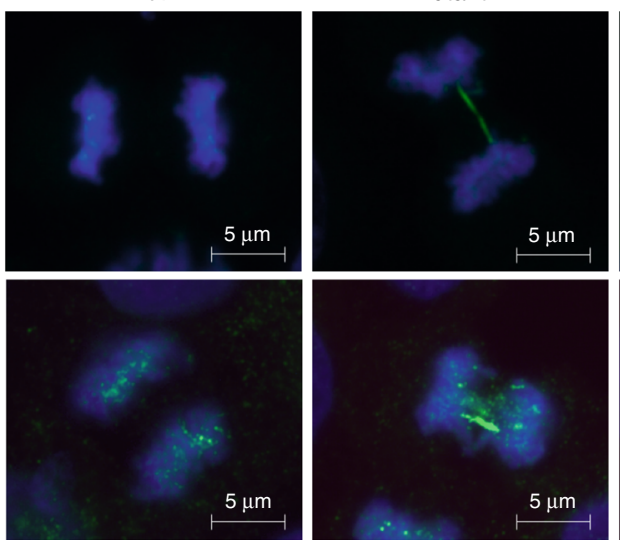

Mutant 1

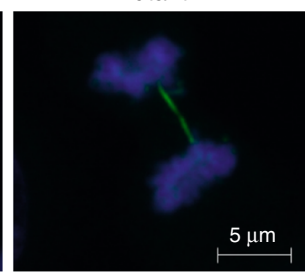

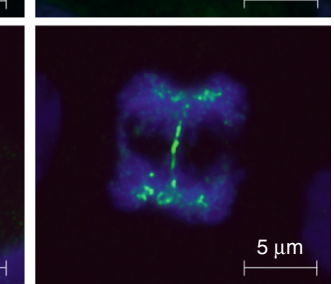

Mutant 2

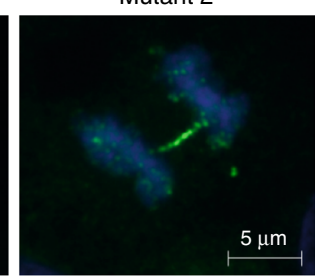

$\mu \mathrm{m}$
C
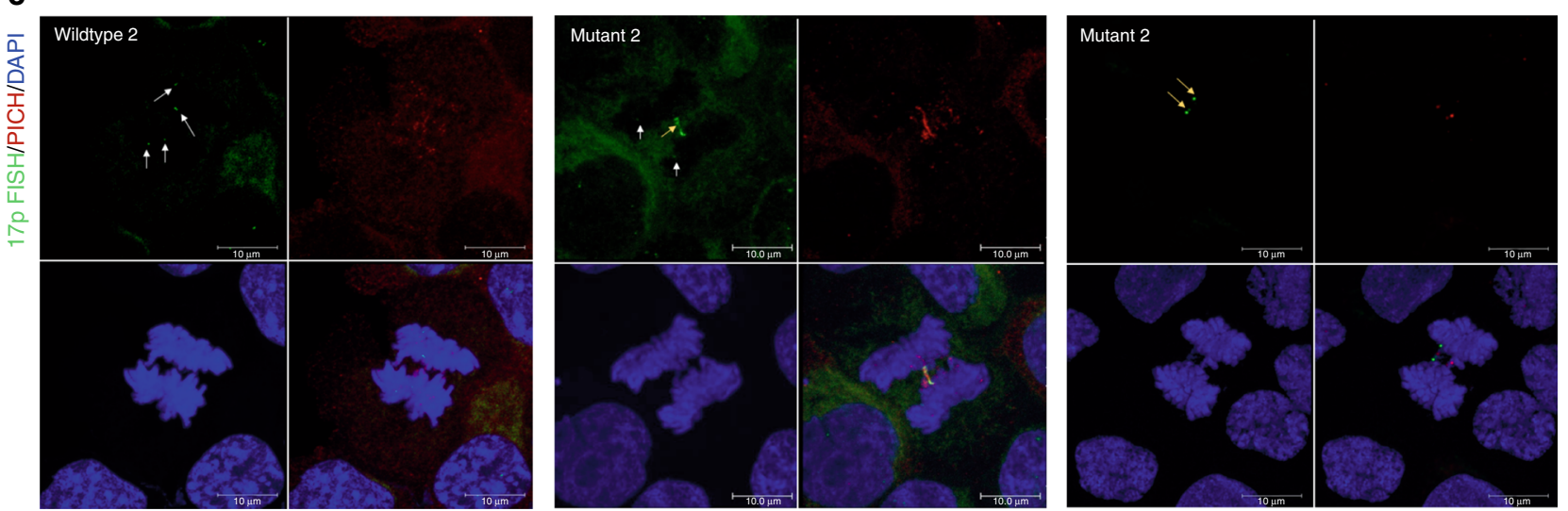

d

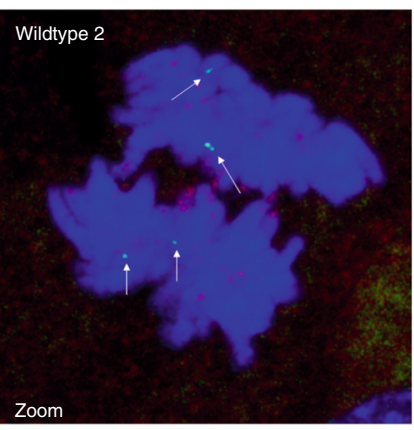

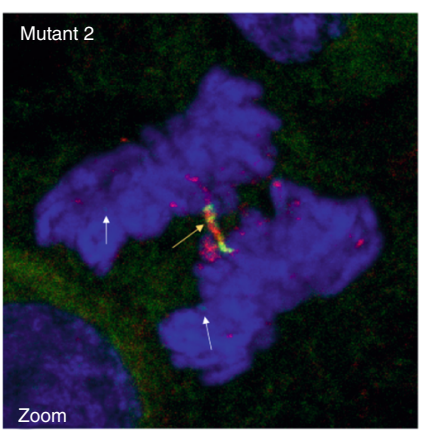

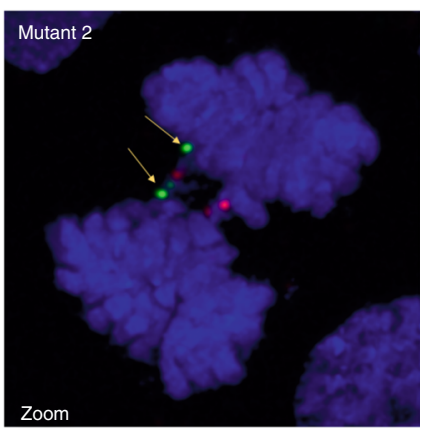

b

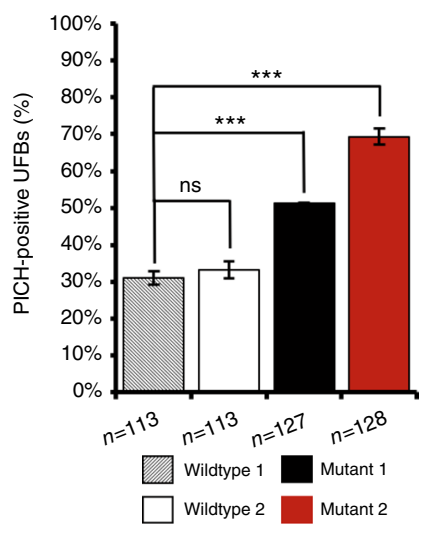

Fig. 4 CTCF site mutant 17p telomere ends form ultra-fine anaphase bridges (UFBs). a Cells were synchronized by thymidine block and collected between 8-9 $\mathrm{h}$ post release and stained with $\mathrm{PICH}$ (green) to detect ultra-fine bridges in anaphase cells. $\mathbf{b}$ Quantification of $\mathbf{a}$. c Cells were treated as in $\mathbf{b}$ and costained for PICH protein and 17p chromosome FISH. Representative image of anaphase bridges for wildtype and Mutant 2. White arrows indicate unaffected 17p FISH signals; yellow arrows indicate 17p FISH signals co-localizing with PICH-positive UFBs. d zoomed fields of merged images in c. e Quantification of $\mathbf{c}$. IF/FISH experiments represent average of three biological replicates and SEM; ${ }^{\star \star} p<0.01$; ${ }^{\star \star \star} p<0.001$, measured by Student's $t$ test

c). On the other hand, aphidicolin treatment did not induce the same extent of sister-telomere loss as was observed with double thymidine block (Supplementary Fig. 4d), perhaps because the thymidine treated cells go through $S$ phase stress twice while aphidicolin is only a single-cell cycle without passage through mitosis. Nevertheless, these findings support the model that subtelomeric CTCF is important for overcoming different forms of replication stress at adjacent telomere repeat DNA.

Increased TERRA transcription rescues defects in $17 p$ CTCF site mutants. To test whether the telomere replication defects were due to CTCF regulation of TERRA transcription and not another CTCF function at the subtelomere, we generated wildtype and mutant clones that could induce TERRA transcription independently of CTCF. Previous studies have shown that fusing TRF1 to the strong transcriptional activation domain of the HSV1 VP16 protein could induce high levels of TERRA ${ }^{39}$. Therefore, we generated wildtype and mutant clones expressing an inducible TRF1-VP16 fusion protein (Fig. 5a). Doxycycline induction of TRF1-VP16 led to a rapid increase in TERRA transcripts in wildtype and mutant cells (Fig. 5b). Remarkably, doxycycline induction of TERRA reduced the appearance of ultra-fine anaphase bridges (Fig. 5c), and reduced sister-telomere loss in mutant CTCF site lines (Fig. 5d). Induction of TRF1 lacking VP16 or VP16 alone in mutant cells did not induce TERRA 
a

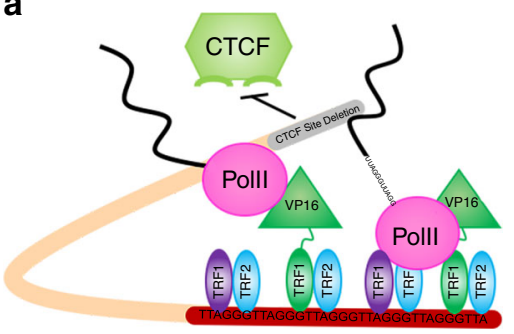

b

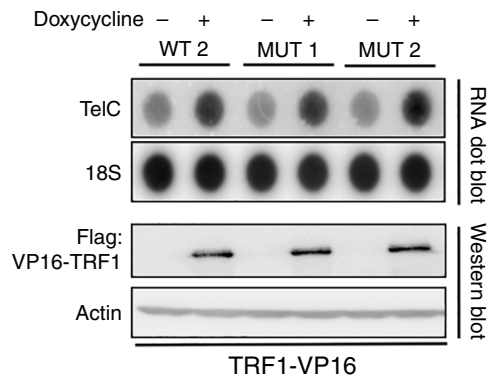

c

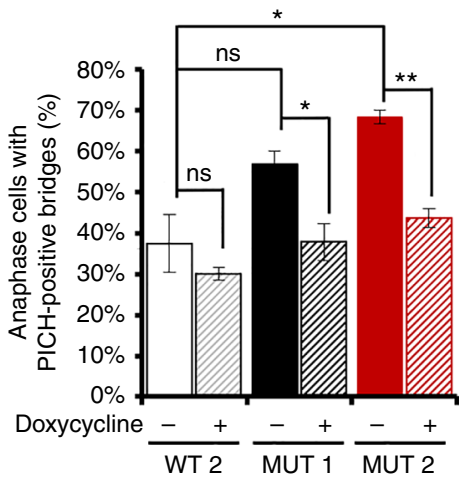

d

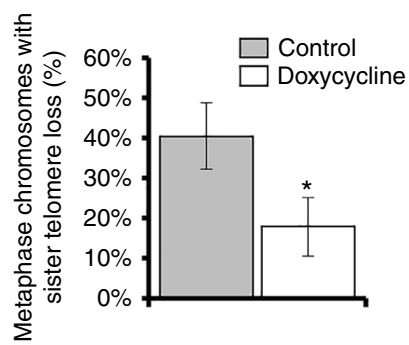

e

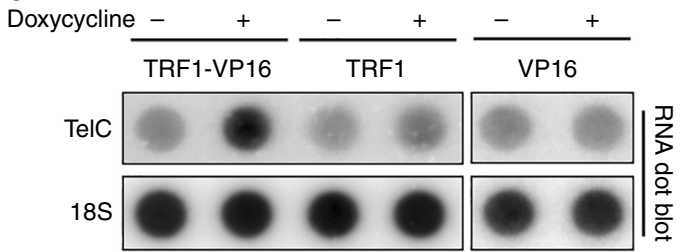

f

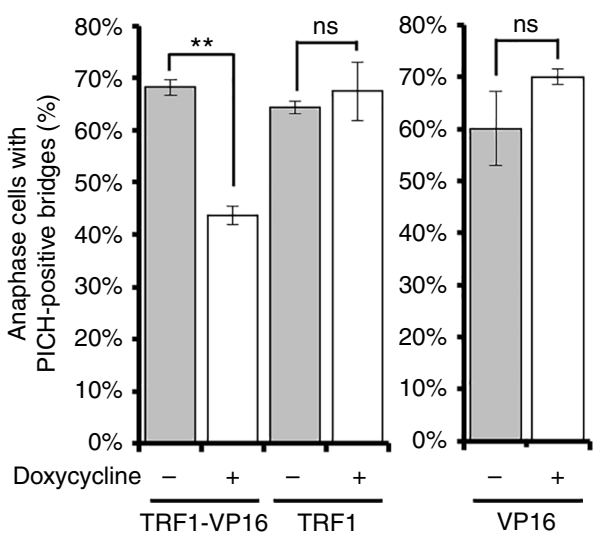

Fig. 5 Increased TERRA transcription rescues mitotic defects in CTCF site mutant cells. Stable mutant and wildtype cell lines expressing a TET-inducible fusion of TRF1-VP16 were selected. Cells were synchronized and expression of the fusion protein was induced $12 \mathrm{~h}$ prior to thymidine release. Cells were collected 8-9 h post release and processed to measure TERRA expression and ultra-fine anaphase bridge formation. a Cartoon of VP16-TRF1-mediated rescue of TERRA transcription. b DotBlot of RNA collected after doxycycline induction in cells expressing the TRF1-VP16 construct in wildtype (WT2), Mutant 1 (Mut 1), or Mutant 2 (Mut 2) cells probed for TelC or $18 \mathrm{~S}$ (top panels). Western blot of TRF1-VP16 and Actin control for same samples (lowerpanels). c Quantification of percentage of cells with PICH-positive anaphase bridges after treatment shown in $\mathbf{b}$. $\mathbf{d}$ Mutant 2 cells treated as in $\mathbf{b}$ were treated with colcemid and collected for metaphase spreads. Sister-telomere loss was quantified on 17p. e Mutant 2 cells expressing inducible TRF1-VP16 fusion were compared to those expressing TRF1 only or VP16 only by RNA dotblot for TERRA and 18S. f UFBs were quantified for cells treated as described in $\mathbf{e}$

expression (Fig. 5e), and did not reduce the number of ultra-fine anaphase bridges (Fig. 5f). These findings indicate that transcriptional activation of TERRA can overcome telomere-specific defects associated with conditions of replication stress.

17p CTCF site mutants exhibit decreased viability and genomic instability. We next tested whether these cell cycle defects lead to any observable phenotypes after cell division. Cells treated with thymidine were analyzed by microscopy at $24 \mathrm{~h}$ after release, $\sim 12$ $\mathrm{h}$ after mitosis. We found that mutant cells exhibited an increased level of micronuclei relative to wildtype cells (Fig. 6a, b). Flow cytometry revealed a corresponding increase in the sub-G0 cell population after thymidine treatment (Fig. 6c), or aphidicolin (Supplementary Fig. 5a) in mutant, but not wildtype cells. Additionally, treatment with replication stress-inducing agents (e.g., thymidine or aphidicolin) induced an increased level of the DNA damage marker $\gamma \mathrm{H} 2 \mathrm{Ax}$ and phospho-p53 in mutant cells similar to wildtype (Supplementary Fig. 5b, c). This indicates that double thymidine block and aphidicolin treatment both induce a persistent DNA damage consistent with the induction of replication stress. Furthermore, mutation of subtelomeric CTCF leads to survivors that exhibit genomic abnormalities, such as micronuclei formation and increased population of SubG1 cells.

\section{Discussion}

Our findings implicate subtelomeric CTCF and TERRA transcription in the proper maintenance of adjacent telomere repeat DNA. CRISPR/Cas9 mutation of the CTCF regulatory element controlling TERRA reveals a potential function for TERRA transcription in promoting the completion of DNA replication at the telomere ends (Fig. 6d, left). Cells lacking subtelomeric CTCF-binding sites were sensitive to replicative stress resulting in mitotic dysfunction with loss of sister-telomere repeat signal and the formation of ultra-fine anaphase bridges (Fig. 6d, right). This sensitivity to replicative stress persists in mutant cells through the rest of the cell cycle and causes decreased viability of sister cells and the formation of micronuclei. Mechanistically, we show that ectopic expression of VP16-TRF1, which increases TERRA 
a
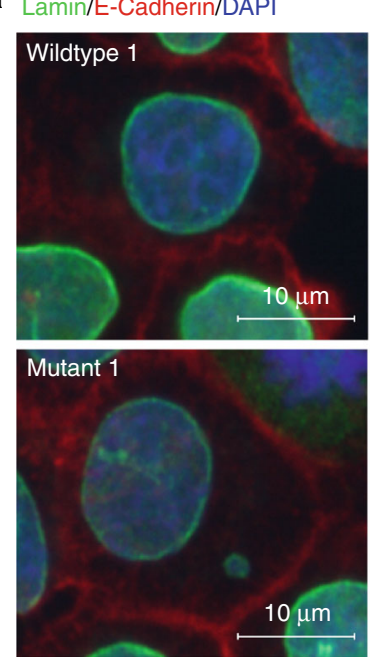

b

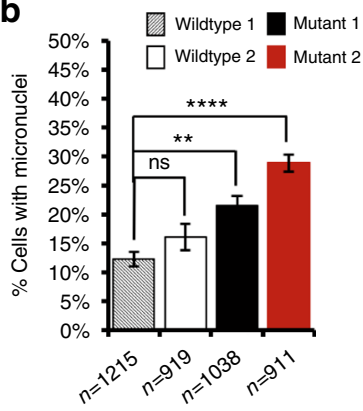

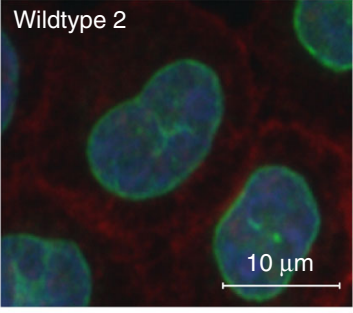

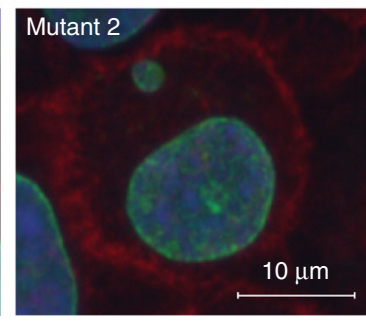

C

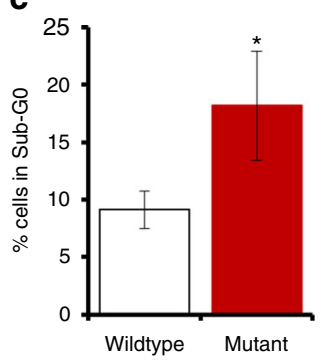

d

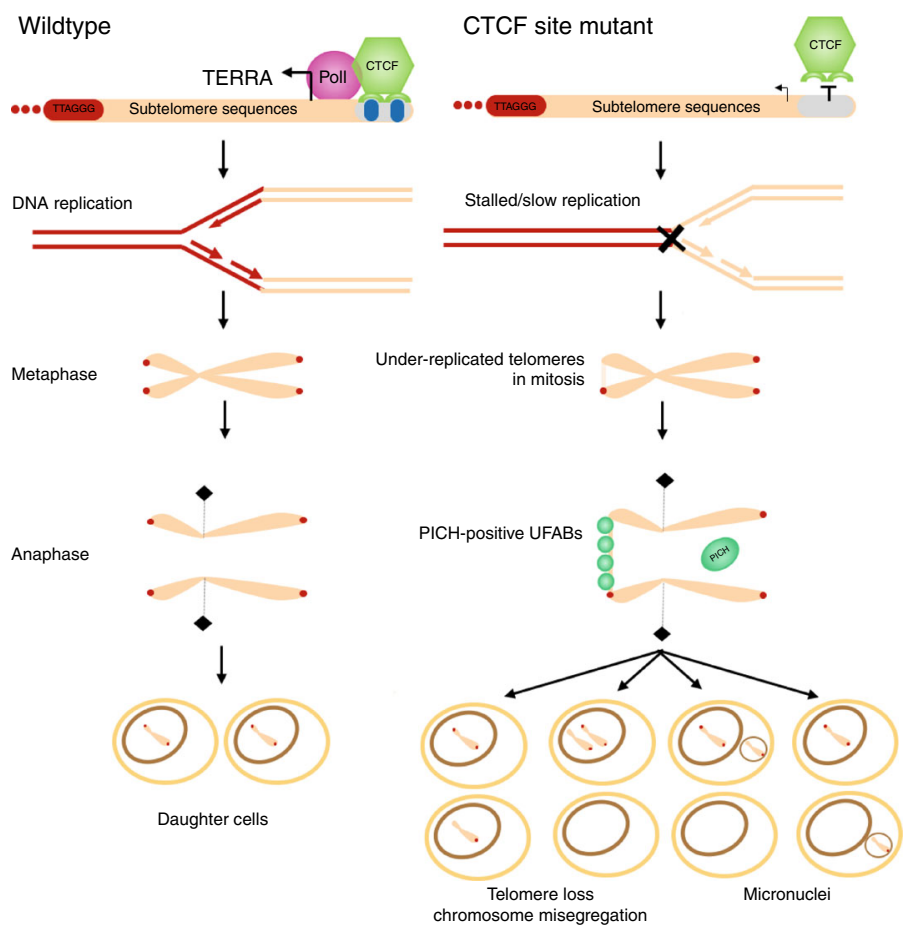

Fig. 6 CTCF site mutant cells exhibit post-mitotic micronuclei and decreased viability. a Wildtype 1 and 2, or Mutant 1 and 2 cells were arrested with thymidine followed by release for $24 \mathrm{~h}$ and then stained with Lamin A/C (nuclear membrane) and E-Cadherin (cell membrane) to assess micronuclei formation after mitosis. Representative images of merged channels for each cell type. $\mathbf{b}$ Quantification of $\mathbf{a}$. All IF/FISH experiments represent average of at least 3 biological replicates and SEM. ${ }^{\star} p<0.05,{ }^{\star \star} p<0.01$, and ${ }^{\star \star \star \star} p<0.0001$, measured by Student $t$ test. c Cells for wildtype 2 and Mutant 2 were collected $24 \mathrm{~h}$ after thymidine release and assessed by flow cytometry for sub-G0 cell population. Data represents average of three independent biological replications and SEM, ${ }^{*} p<0.05$, by $t$ test. $\mathbf{d}$ Schematic depicting effect of CTCF-mediated TERRA transcriptional on telomere replication and mitosis

transcription, can protect against the formation of PICH-positive anaphase bridges. We propose that CTCF-driven TERRA transcription initiating within the subtelomere facilitates the completion of DNA replication of adjacent telomere repeats.

TERRA transcription may enhance telomere maintenance by overcoming some of many telomere-specific challenges to DNA replication ${ }^{40}$. Telomere transcription and TERRA molecules may facilitate DNA replication by promoting the dissociation of shelterin components that may otherwise obstruct replication fork progression ${ }^{41}$. Telomeres typically replicate unidirectionally ${ }^{42}$ and late in the cell cycle ${ }^{43,44}$ and cell cycle delays due to replication stress are likely to lead to a loss of telomere repeat DNA. TERRA transcription may advance the replication timing of telomere repeat regions ${ }^{45}$, and therefore increase the probability of completing DNA replication. Thus, TERRA transcription may facilitate telomere DNA replication by multiple mechanisms.

TERRA may also have complex regulatory functions at telomeres. TERRA has been implicated in the formation of R-loops ${ }^{23}$, and G-quadruplex structures in the telomere repeats ${ }^{46}$, structures which may inhibit, as well as promote telomere DNA replication. R-loop structures may act as a barrier to replication machinery ${ }^{20}$, 22 , although there are normal cell pathways that promote bypass of R-loop structures. In contrast, G-quadruplex structures, suggested to form on the opposing strand of the R-loop, have been shown to support origin binding and replication initiation, which could be utilized as a means to initiate replication from telomere repeats or rescue a stalled fork heading into the repeats from a subtelomeric origin ${ }^{47,48}$.

In addition to TERRA transcription, CTCF may facilitate telomere DNA replication by altering chromatin structure. The CTCF-binding site is required for active histone $\mathrm{H} 3 \mathrm{~K} 4 \mathrm{me} 3$ enrichment (Fig. 2), and may prevent the formation of heterochromatin that could otherwise further impede DNA replication through telomeres. The $17 \mathrm{p}$ family of subtelomeric CTCFbinding motifs have structural similarity to the tandem copies that have been implicated in transcriptional regulatory function $^{35}$. These type of motifs have been found to be enriched at other repetitive elements ${ }^{49}$, suggesting that CTCF plays an important regulatory role at repetitive elements. CTCF-binding sites show significant genetic variation in tumor tissues, and some of these variations have been implicated in altered transcription control in cancer cells $s^{50-53}$. However, we have been unable to identify any significant variations in the $17 \mathrm{p}$ family of subtelomeric CTCF-binding sites from the TCGA database or 1000 genomes, suggesting that these CTCF sites may be essential for human cell viability.

Finally, it has been suggested that the majority of TERRA transcripts arise from a single subtelomere and interact in trans with all the telomere ends ${ }^{18}$. Our data does not exclude the possibility that large quantities of TERRA are generated from a single chromosome in some cell types, nor that TERRA 
transcripts can interact in trans with other telomere ends. However, our data strongly suggest that CTCF-binding site mutation and decreased levels of TERRA transcription directly impacts the DNA replication efficiency of the same source telomere end from which it is transcribed, indicating that it has a direct cis-acting effect on telomere stability. In conclusion, the data presented here supports a function for CTCF-mediated TERRA transcription in maintaining proper telomere replication and chromosome stability.

\section{Methods}

Cell culture and treatments. HCT116 cells (purchased from ATCC) were cultured in DMEM with 10\% FBS and penicillin/streptomycin. Clones expressing VP16 constructs were cultured in 10\% tet-free FBS (Sigma) and G-418. Cells were in $2.5 \mathrm{mM}$ thymidine and $0.2 \mu \mathrm{M}$ aphidicolin, and mitotic cells were collected in $0.1 \mu \mathrm{g} / \mathrm{mL}$ colcemid. For aphidicolin experiments and BrdU-immunoprecipitation assays, Cdk1 inhibitor, RO-3306, was used at a dose of $9 \mu \mathrm{M}$ to inhibit cells from entering mitosis. For BrdU-immunoprecipitation assays and BrdU was added at 10 $\mu \mathrm{M}$ at indicated time points.

Vectors and cloning. Cas9/guide RNA dual vectors were constructed using sequences for 17 p-targeting gRNA (Supplementary Table 3$)^{54}$. Homology blocks were constructed from subtelomere sequences and synthesized by IDT Inc, sequences are also listed in Supplementary Table 4. To construct the TRF1-VP16 constructs, TRF1 $\Delta \mathrm{N}$ (44-439), and VP16-TRF1 $\Delta \mathrm{N}$ (44-439) were sub-cloned into the pINDUCER20 vector ${ }^{55}$ from plasmids for TERRA induction ${ }^{39}$. Briefly, the DNA fragments were introduced into the pENTR Directional TOPO Cloning vector (Invitrogen) as per the manufacturer's instructions, and followed by the Gateway cloning into the pINDUCER20 vector using the LR Clonase II (Invitrogen).

CRISPR cell-line construction. Homology blocks were amplified by PCR and cotransfected into cells with Cas 9 constructs. Cells were clonally selected with puromycin for 10-14 days. Genomic DNA was isolated from all clones, and mutations were validated by PCR and restriction digest. TRF1-VP16 lines were constructed by lentiviral introduction of vectors. Lentivirus was produced from $293 \mathrm{~T}$ cells by co-transfecting the constructs with viral packaging vectors pVSVG, pRSV, and pMDL. All the cultured media were supplemented with $10 \%$ (vol/vol) tetracyclinfree FBS (Corning) and harvested at $48 \mathrm{~h}$ after transfection. To generate the inducible cell lines, $1 \times 10^{6}$ HCT116 cells were infected with $1 \mathrm{~mL}$ lentivirus overnight in the presence of $2 \mu \mathrm{g} / \mathrm{mL}$ Polybrene (Sigma). Infected cells were selected by $1 \mathrm{mg} / \mathrm{mL}$ G418 $48 \mathrm{~h}$ after infection. Cells were stably selected for 6 days in the tetracyclin-free medium prior to use in experiments. For TERRA induction, $0.5 \mu \mathrm{g} / \mathrm{mL}$ Doxycycline was added to the medium $12 \mathrm{~h}$ prior to thymidine release in synchronized experiments.

Chromatin immunoprecipitation. Cells were fixed and processed for chromatin immunoprecipitation ${ }^{9}$. Briefly, cells were fixed with formaldehyde for $15 \mathrm{~min}$, washed, and lysed in SDS Lysis buffer. Chromatin lysates were sonicated for five cycles, $10 \mathrm{~min}$ each, $30 \mathrm{~s}$ on/off. Lysates were diluted and pre-cleared with protein A sepharose and bacterial tRNA for blocking. Lysates were immunoprecipitated with indicated antibodies overnight. Antibodies ( $2 \mu \mathrm{g}$ of each) used were as follows: anti-CTCF, EMD Millipore 07-729; anti-H3, Active Motif \#39163; and antiH3K4me3, EMD Millipore 07-473. Immune complexes were collected with protein A sepharose for $2 \mathrm{~h}$, beads were washed, and bound DNA was collected, and then purified with PCR purification kit (Sigma). Protein binding was determined by qPCR or DNA dotblot. Primers used in qPCR assessment are listed in Supplementary Table 1 and locations of $17 \mathrm{p}$ primers are shown visually in Supplementary Fig. 1a.

RT-qPCR and RNA DotBlot. RNA was isolated using Trizol (Invitrogen) following manufacture's instructions. An aliquot of $5 \mu \mathrm{g}$ of RNA was blotted onto membrane for dot blots ${ }^{9}$. RNA was DNase treated and converted to random primed cDNA using Superscript III kit (Invitrogen). cDNA was assessed for TERRA transcription levels, using GAPDH as control. Primers for qPCR analysis are listed in Supplementary Table 2 and locations of 17p primers are shown visually in Supplementary Fig. 1a.

Brdu immunoprecipitation. Genomic DNA was collected from cells after incorporation of BrdU. DNA isolation kit (Promega). DNA was sonicated to between $100-500 \mathrm{bp}$ in length. Sonicated DNA was denatured for $5 \mathrm{~min}$ at $100 \mathrm{C}$ and diluted in IP buffer (10 mM Sodium Phosphate pH7, 0.14 M NaCl, 0.05\% Triton X$100)$. BrdU containing DNA was immunoprecipitated with $2-4 \mu \mathrm{g}$ of anti-BrdU antibody (BD Pharmingin mouse-anti BrdU Clone $44(\# 347850)$ ) for $1 \mathrm{~h}$ at room temperature and collected using protein $\mathrm{G}$ agarose beads for $30 \mathrm{~min}$. Isolated DNA/ immune complexes were washed 4 times with IP buffer and dissociated using BrdU
IP lysis buffer (10 mM EDTA, $20 \mathrm{mM}$ Tris pH 8.8, 0.5\% SDS, $0.25 \mathrm{mg} / \mathrm{mL}$ Proteinase K) overnight. DNA was purified by column purification (Sigma). BrdU incorporation into genomic regions was assessed by qPCR.

Immunofluorescence and DNA-FISH. For non-metaphase cell preparations, cells were collected by trypsinization and counted. $1 \times 10^{5}$ cells were spun onto $10 \mathrm{~mm}$ coverslips for processing. Fixation was done with either 1\% PFA for immunofluorescence only or 50:50 mix methanol:acetone for IF-FISH. IF-only cells were extracted with $0.5 \% \mathrm{NP}-40$ for $10 \mathrm{~min}$ and probed with primary (1:300) and secondary antibodies (1:500). Primary antibodies for PICH were from Abnova (H00054821-B01P) or EMD Millipore (04-1540); E-Cadherin, Abcam (ab53033); and Lamin A/C, DSHB (MANLAC1(4A7). Secondary Antibodies; Goat antimouse/anti-rabbit AlexaFluor-488 (Thermo Fisher, A-11029 and A-1134) and AlexaFluor-594 (Thermo Fisher, A-11032 and A-11037) were used. Cells were counterstained with DAPI and imaged using a Leica DMRE upright microscope.

IF-FISH cells were stained with primary and secondary antibodies after fixation and then post-fixed with $2 \% \mathrm{PFA}+1 \%$ TritonX100. The primary antibodies for PICH used was anti-PICH, Abnova (H00054821-B01P, 1:100). Secondary antimouse AlexaFluor-594 (Thermo Fisher, A-11032, 1:500). Cells were washed with PBS, then extracted with $0.2 \mathrm{~N} \mathrm{HCL} / 0.02 \%$ TritonX100 for $10 \mathrm{~min}$ at $4 \mathrm{C}$. The cells were dehydrated with ethanol series and probed with FISH probe (Cytocell LPT17pG) overnight at $37 \mathrm{C}$. Coverslips were washed $2 \times$ with Wash 1 (70\% Formamide, $10 \mathrm{mM}$ Tris pH7-7.5, 0.1\% BSA), 3× Wash $2(0.1 \mathrm{M}$ Tris pH7-7.5, $0.15 \mathrm{M} \mathrm{NaCl}, 0.08 \%$ Tween), counterstained with DAPI, and mounted on slides for imaging. The slides were imaged with Leica TCS SP5 II scanning laser confocal microscope. Anaphase cells were imaged as a $\mathrm{Z}$ stack and representative images are Maximum Projections of all stacks.

Mitotic cells were collected by trypsinization and swelled in $0.025 \mathrm{M} \mathrm{KCl}$ for 25 min at $37 \mathrm{C}$. Cells were fixed with 3 resuspensions of a methanol: acetic acid, 3:1 mix, and stored in fixative at least $24 \mathrm{~h}$ prior to dropping. Mitotic cells were dropped onto chilled clean glass slides and dried at least $4 \mathrm{~h}$ prior to staining. After drying, the cells were fixed for $5 \mathrm{~min}$ with $1 \%$ paraformaldehyde in PBS. Cells were washed and then dehydrated with ethanol wash series. Cells were co-stained with $17 \mathrm{p}$ subtelomere probe (Cytocell LPT17pG or LPT17pR) and telomere repeat (CCCTAA) PNA probe, TelC-TMR or telomere repeat (GGGATT) PNA probe, and TelG-FAM (Panagene) in Hybridization Buffer B (Cytocell) overnight at $37 \mathrm{C}$. Slides were washed as described above with Wash 1 and 2 , and counterstained with DAPI. Slides were dehydrated with another ethanol series and mounted with Slow Anti Fade Gold mounting solution (Invitrogen). Mounted Slides were imaged on Leica DMRE upright microscope.

Data availability. All data presented in this manuscript is available from the authors.

Received: 26 March 2017 Accepted: 14 November 2017 Published online: 13 December 2017

\section{References}

1. Greider, C. W. Regulating telomere length from the inside out: the replication fork model. Genes Dev. 30, 1483-1491 (2016).

2. Wu, R. A., Upton, H. E., Vogan, J. M. \& Collins, K. Telomerase mechanism of telomere synthesis. Annu. Rev. Biochem. 86, 439-460 (2017).

3. Martinez, P. \& Blasco, M. A. Replicating through telomeres: a means to an end. Trends Biochem. Sci. 40, 504-515 (2015).

4. Palm, W. \& de Lange, T. How shelterin protects mammalian telomeres. Annu. Rev. Genet. 42, 301-334 (2008).

5. de Lange, T. Shelterin: the protein complex that shapes and safeguards human telomeres. Genes Dev. 19, 2100-2110 (2005).

6. Mefford, H. C. \& Trask, B. J. The complex structure and dynamic evolution of human subtelomeres. Nat. Rev. Genet. 3, 91-102 (2002).

7. Riethman, H., Ambrosini, A. \& Paul, S. Human subtelomere structure and variation. Chromosome Res. 13, 505-515 (2005).

8. Stong, N. et al. Subtelomeric CTCF and cohesin binding site organization using improved subtelomere assemblies and a novel annotation pipeline. Genome Res. 24, 1039-1050 (2014).

9. Deng, Z. et al. A role for CTCF and cohesin in subtelomere chromatin organization, TERRA transcription, and telomere end protection. EMBO J. $\mathbf{3 1}$ 4165-4178 (2012).

10. Tutton, S. et al. Subtelomeric p53 binding prevents accumulation of DNA damage at human telomeres. EMBO J. 35, 193-207 (2016).

11. Schoeftner, S. \& Blasco, M. A. A 'higher order' of telomere regulation: telomere heterochromatin and telomeric RNAs. EMBO J. 28, 2323-2336 (2009).

12. Blasco, M. A. The epigenetic regulation of mammalian telomeres. Nat. Rev. Genet. 8, 299-309 (2007).

13. Luke, B. \& Lingner, J. TERRA: telomeric repeat-containing RNA. EMBO J. 28, 2503-2510 (2009). 
14. Luke, B. et al. The Ratlp 5' to 3' exonuclease degrades telomeric repeatcontaining RNA and promotes telomere elongation in Saccharomyces cerevisiae. Mol. Cell 32, 465-477 (2008).

15. Schoeftner, S. \& Blasco, M. A. Developmentally regulated transcription of mammalian telomeres by DNA-dependent RNA polymerase II. Nat. Cell Biol. 10, 228-236 (2008).

16. Arora, R. et al. RNaseH1 regulates TERRA-telomeric DNA hybrids and telomere maintenance in ALT tumour cells. Nat. Commun. 5, 5220 (2014).

17. Redon, S., Zemp, I. \& Lingner, J. A three-state model for the regulation of telomerase by TERRA and hnRNPA1. Nucleic Acids Res. 41, 9117-9128 (2013).

18. Montero, J. J., Lopez de Silanes, I., Grana, O. \& Blasco, M. A. Telomeric RNAs are essential to maintain telomeres. Nat. Commun. 7, 12534 (2016).

19. Lopez de Silanes, I. et al. Identification of TERRA locus unveils a telomere protection role through association to nearly all chromosomes. Nat. Commun. 5, 4723 (2014)

20. Aguilera, A. \& Garcia-Muse, T. R loops: from transcription byproducts to threats to genome stability. Mol. Cell 46, 115-124 (2012).

21. Balk, B. et al. Telomeric RNA-DNA hybrids affect telomere-length dynamics and senescence. Nat. Struct. Mol. Biol. 20, 1199-1205 (2013).

22. Gan, W. et al. R-loop-mediated genomic instability is caused by impairment of replication fork progression. Genes Dev. 25, 2041-2056 (2011).

23. Pfeiffer, V., Crittin, J., Grolimund, L. \& Lingner, J. The THO complex component Thp2 counteracts telomeric R-loops and telomere shortening. EMBO J. 32, 2861-2871 (2013).

24. Yu, T. Y., Kao, Y. W. \& Lin, J. J. Telomeric transcripts stimulate telomere recombination to suppress senescence in cells lacking telomerase. Proc. Natl Acad. Sci. USA 111, 3377-3382 (2014).

25. Porro, A. et al. Functional characterization of the TERRA transcriptome at damaged telomeres. Nat. Commun. 5, 5379 (2014).

26. Flynn, R. L. et al. TERRA and hnRNPA1 orchestrate an RPA-to-POT1 switch on telomeric single-stranded DNA. Nature 471, 532-536 (2011).

27. Arnoult, N., Van Beneden, A. \& Decottignies, A. Telomere length regulates TERRA levels through increased trimethylation of telomeric $\mathrm{H} 3 \mathrm{~K} 9$ and HP1alpha. Nat. Struct. Mol. Biol. 19, 948-956 (2012).

28. Porro, A., Feuerhahn, S., Reichenbach, P. \& Lingner, J. Molecular dissection of telomeric repeat-containing RNA biogenesis unveils the presence of distinct and multiple regulatory pathways. Mol. Cell Biol. 30, 4808-4817 (2010).

29. Episkopou, H. et al. Alternative lengthening of Telomeres is characterized by reduced compaction of telomeric chromatin. Nucleic Acids Res. 42, 4391-4405 (2014).

30. Lovejoy, C. A. et al. Loss of ATRX, genome instability, and an altered DNA damage response are hallmarks of the alternative lengthening of telomeres pathway. PLoS Genet. 8, e1002772 (2012).

31. Ng, L. J., Cropley, J. E., Pickett, H. A., Reddel, R. R. \& Suter, C. M. Telomerase activity is associated with an increase in DNA methylation at the proximal subtelomere and a reduction in telomeric transcription. Nucleic Acids Res. 37, 1152-1159 (2009)

32. Nergadze, S. G. et al. CpG-island promoters drive transcription of human telomeres. RNA 15, 2186-2194 (2009).

33. Ong, C. T. \& Corces, V. G. CTCF: an architectural protein bridging genome topology and function. Nat. Rev. Genet. 15, 234-246 (2014).

34. Denker, A. \& de Laat, W. The second decade of 3C technologies: detailed insights into nuclear organization. Genes Dev. 30, 1357-1382 (2016).

35. Pugacheva, E. M. et al. Comparative analyses of CTCF and BORIS occupancies uncover two distinct classes of CTCF binding genomic regions. Genome Biol. 16, 161 (2015)

36. Minocherhomji, S. et al. Replication stress activates DNA repair synthesis in mitosis. Nature 528, 286-290 (2015)

37. Chan, K. L., Palmai-Pallag, T., Ying, S. \& Hickson, I. D. Replication stress induces sister-chromatid bridging at fragile site loci in mitosis. Nat. Cell. Biol. 11, 753-760 (2009).

38. Liu, Y., Nielsen, C. F., Yao, Q. \& Hickson, I. D. The origins and processing of ultra fine anaphase DNA bridges. Curr. Opin. Genet. Dev. 26, 1-5 (2014).

39. Wang, Z. et al. Telomeric repeat-containing RNA (TERRA) constitutes a nucleoprotein component of extracellular inflammatory exosomes. Proc. Natl Acad. Sci. USA 112, E6293-E6300 (2015).

40. Sfeir, A. et al. Mammalian telomeres resemble fragile sites and require TRF1 for efficient replication. Cell 138, 90-103 (2009).

41. Nera, B., Huang, H. S., Lai, T. \& Xu, L. Elevated levels of TRF2 induce telomeric ultrafine anaphase bridges and rapid telomere deletions. Nat. Commun. 6 , 10132 (2015).

42. Drosopoulos, W. C., Kosiyatrakul, S. T., Yan, Z., Calderano, S. G. \& Schildkraut, C. L. Human telomeres replicate using chromosome-specific, rather than universal, replication programs. J. Cell Biol. 197, 253-266 (2012).

43. Arnoult, N. et al. Replication timing of human telomeres is chromosome armspecific, influenced by subtelomeric structures and connected to nuclear localization. PLoS Genet. 6, e1000920 (2010).
44. Piqueret-Stephan, L., Ricoul, M., Hempel, W. M. \& Sabatier, L. Replication timing of human telomeres is conserved during immortalization and influenced by respective subtelomeres. Sci. Rep. 6, 32510 (2016).

45. Rivera-Mulia, J. C. \& Gilbert, D. M. Replication timing and transcriptional control: beyond cause and effect-part III. Curr. Opin. Cell. Biol. 40, 168-178 (2016).

46. Xu, Y., Suzuki, Y., Ito, K. \& Komiyama, M. Telomeric repeat-containing RNA structure in living cells. Proc. Natl Acad. Sci. USA 107, 14579-14584 (2010).

47. Hoshina, S. et al. Human origin recognition complex binds preferentially to Gquadruplex-preferable RNA and single-stranded DNA. J. Biol. Chem. 288, 30161-30171 (2013).

48. Lombrana, R., Almeida, R., Alvarez, A. \& Gomez, M. R-loops and initiation of DNA replication in human cells: a missing link? Front. Genet. 6, 158 (2015).

49. Pugacheva, E. M. et al. The cancer-associated CTCFL/BORIS protein targets multiple classes of genomic repeats, with a distinct binding and functional preference for humanoid-specific SVA transposable elements. Epigenetics Chromatin 9, 35 (2016).

50. Kaiser, V. B., Taylor, M. S. \& Semple, C. A. Mutational biases drive elevated rates of substitution at regulatory sites across cancer types. PLoS Genet. 12, e1006207 (2016).

51. Katainen, R. et al. CTCF/cohesin-binding sites are frequently mutated in cancer. Nat. Genet. 47, 818-821 (2015).

52. Poulos, R. C. et al. Functional mutations form at CTCF-cohesin binding sites in melanoma due to uneven nucleotide excision repair across the motif. Cell Rep. 17, 2865-2872 (2016).

53. Umer, H. M. et al. A significant regulatory mutation burden at a high-affinity position of the CTCF motif in gastrointestinal cancers. Hum. Mutat. 37, 904-913 (2016).

54. Ran, F. A. et al. Genome engineering using the CRISPR-Cas9 system. Nat. Protoc. 8, 2281-2308 (2013).

55. Meerbrey, K. L. et al. The pINDUCER lentiviral toolkit for inducible RNA interference in vitro and in vivo. Proc. Natl Acad. Sci. USA 108, 3665-3670 (2011).

\section{Acknowledgements}

We thank Harold Riethman for discussion and advice on telomere and subtelomere biology. Special thanks to J. Faust, J. Hayden, and F. Keeney of the Wistar Flow Cytometry and Imaging Facilities. K.B. was supported by a Wistar-NRSA sponsored Cance Research Training Program Fellowship (T32 CA09171). This research is supported by funds from the National Cancer Institute RO1 CA140652 to PML, and the Wistar Institute Cancer Center P30 CA010815.

\section{Author contributions}

Conception and experimental design: K.B. and P.M.L. Methodology and data acquisition: K.B., O.V., S.T., Z.D., and Z.W. Analysis and interpretation of data: K.B. and P.M.L Manuscript writing: K.B. and P.M.L.

\section{Additional information}

Supplementary Information accompanies this paper at https://doi.org/10.1038/s41467017-02212-w.

Competing interests: The authors declare no competing financial interests.

Reprints and permission information is available online at http://npg.nature.com/ reprintsandpermissions/

Publisher's note: Springer Nature remains neutral with regard to jurisdictional claims in published maps and institutional affiliations.

Open Access This article is licensed under a Creative Commons Attribution 4.0 International License, which permits use, sharing, adaptation, distribution and reproduction in any medium or format, as long as you give appropriate credit to the original author(s) and the source, provide a link to the Creative Commons license, and indicate if changes were made. The images or other third party material in this article are included in the article's Creative Commons license, unless indicated otherwise in a credit line to the material. If material is not included in the article's Creative Commons license and your intended use is not permitted by statutory regulation or exceeds the permitted use, you will need to obtain permission directly from the copyright holder. To view a copy of this license, visit http://creativecommons.org/ licenses/by/4.0/.

(C) The Author(s) 2017 